Bull. Korean Math. Soc. 46 (2009), No. 5, pp. 845-856

DOI 10.4134/BKMS.2009.46.5.845

\title{
PATH-CONNECTED AND NON PATH-CONNECTED ORTHOMODULAR LATTICES
}

\author{
Eunsoon Park And Wonhee Song
}

\begin{abstract}
A block of an orthomodular lattice $L$ is a maximal Boolean subalgebra of $L$. A site is a subalgebra of an orthomodular lattice $L$ of the form $S=A \cap B$, where $A$ and $B$ are distinct blocks of $L$. An orthomodular lattice $L$ is called with finite sites if $|A \cap B|<\infty$ for all distinct blocks $A, B$ of $L$. We prove that there exists a weakly path-connected orthomodular lattice with finite sites which is not path-connected and if $L$ is an orthomodular lattice such that the height of the join-semilattice $[C o m L]_{\vee}$ generated by the commutators of $L$ is finite, then $L$ is pathconnected.
\end{abstract}

\section{Introduction}

A path of an orthomodular lattice was defined by Bruns [1] and has been studied by several authors. We have the following classes of path-connected orthomodular lattices: every block-finite orthomodular lattice is path-connected [1], and every commutator-finite orthomodular lattice is path-connected [2], and every vertex-finite orthomodular lattice is path-connected [7]. We study some conditions such that an irreducible orthomodular lattice is to be simple, and some properties of paths of an orthomodular lattice has been used to prove that every block-finite irreducible orthomodular lattice is simple [9], and every vertex-finite irreducible orthomodular lattice is simple [7]. In this paper, we extend these results, and find some path-connected orthomodular lattices and some properties of path-connected orthomodular lattices.

An orthomodular poset (abbreviated by OMP) is a partially ordered set $P$ which satisfies the orthomodular law: if $x \leq y$, then $y=x \vee\left(x^{\prime} \wedge y\right)[6]$. An orthomodular lattice (abbreviated by OML) is an ortholattice $L$ which satisfies the orthomodular law [6]. A Boolean algebra $B$ is an ortholattice satisfying the distributive law: $x \vee(y \wedge z)=(x \vee y) \wedge(x \vee z) \forall x, y, z \in B$.

Received June 30, 2008; Revised January 21, 2009.

2000 Mathematics Subject Classification. 06C15.

Key words and phrases. orthomodular lattice, with finite sites, path-connected, non pathconnected, Boolean algebra.

This research was supported by the Soongsil University Research Fund. 
A subalgebra of an OML $L$ is a nonempty subset $M$ of $L$ which is closed under the operations $\vee, \wedge$ and ' . We write $M \leq L$ if $M$ is a subalgebra of $L$. If $M \leq L$ and $a, b \in M$ with $a \leq b$, then the relative interval sublattice $M[a, b]=$ $\{x \in M \mid a \leq x \leq b\}$ is an OML with the relative orthocomplementation $\sharp$ on $M[a, b]$ given by $c^{\sharp}=\left(a \vee c^{\prime}\right) \wedge b=a \vee\left(c^{\prime} \wedge b\right) \quad \forall c \in M[a, b]$. In particular, $L[a, b]$ will be denoted by $[a, b]$ if there is no ambiguity.

The commutator of $a$ and $b$ of an OML $L$ is denoted by $a * b$, and is defined by $a * b=(a \vee b) \wedge\left(a \vee b^{\prime}\right) \wedge\left(a^{\prime} \vee b\right) \wedge\left(a^{\prime} \vee b^{\prime}\right)$. The set of all commutators of $L$ is denoted by $C o m L$ and $L$ is said to be commutator-finite if $|C o m L|$ is finite. For elements $a, b$ of an OML, we say $a$ commutes with $b$, in symbols $a \mathbf{C} b$, if $a * b=0$. If $M$ is a subset of an OML $L$, the set $\mathbf{C}(M)=\{x \in L \mid x \mathbf{C} m \quad \forall m \in M\}$ is called the commutant of $M$ in $L$ and the set $\mathbf{C e n}(M)=\mathbf{C}(M) \cap M$ is called the center of $M$. The set $\mathbf{C}(L)$ is called the center of $L$ and then $\mathbf{C}(L)=\bigcap\{\mathbf{C}(a) \mid a \in L\}$. An OML $L$ is called irreducible if $\mathbf{C}(L)=\{0,1\}$, and $L$ is called reducible if it is not irreducible.

$A$ block of an OML $L$ is a maximal Boolean subalgebra of $L$. The set of all blocks of $L$ is denoted by $\mathfrak{A}_{L}$. Note that $\bigcup \mathfrak{A}_{L}=L$ and $\bigcap \mathfrak{A}_{L}=\mathbf{C}(L)$. An OML $L$ is said to be block-finite if $\left|\mathfrak{A}_{L}\right|$ is finite.

For any $e$ in an OML $L$, the subalgebra $S_{e}=\left[0, e^{\prime}\right] \cup[e, 1]$ is called the (principal) section generated by $e$. Note that for $A, B \in \mathfrak{A}_{L}$, if $e \in A \cap B$ and $A \cap B=S_{e} \cap(A \cup B)$, then $A \cap B=S_{e} \cap A=S_{e} \cap B$ [1].

Definition 1.1. For blocks $A, B$ of an OML $L$ define $A \stackrel{w k}{\sim} B$ if and only if $A \cap B=S_{e} \cap(A \cup B)$ for some $e \in A \cap B ; A \sim B$ if and only if $A \neq B$ and $A \cup B \leq L ; A \approx B$ if and only if $A \sim B$ and $A \cap B \neq \mathbf{C}(L)$.

$A$ path in $L$ is a finite sequence $B_{0}, B_{1}, \ldots, B_{n}(n \geq 0)$ in $\mathfrak{A}_{L}$ satisfying $B_{i} \sim B_{i+1}$ whenever $0 \leq i<n$. The path is said to join the blocks $B_{0}$ and $B_{n}$. The number $n$ is said to be the length of the path. A path is said to be proper if and only if $n=1$ or $B_{i} \approx B_{i+1}$ holds whenever $0 \leq i<n$. A path is called to be strictly proper if and only if $B_{i} \approx B_{i+1}$ holds whenever $0 \leq i<n[1]$.

Let $A, B$ be two blocks of an OML $L$. If $A \sim B$ holds, then there exists a unique element $e \in A \cap B$ satisfying $A \cap B=(A \cup B) \cap S_{e}$ [1]. Using this element $e$, we say that $A$ and $B$ are linked at $e$ (strongly linked at $e$ ) if $A \sim B$ $(A \approx B)$, and use the notation $A \sim_{e} B\left(A \approx_{e} B\right)$. The element $e$ is called $a$ vertex of $L$ and it is the commutator of any $x \in A \backslash B$ and $y \in B \backslash A$ [1]. The set of all vertices of $L$ is denoted by $V_{L}$ and $L$ is said to be vertex-finite if $\left|V_{L}\right|$ is finite.

Note that $A \approx B$ implies $A \sim B$, and $A \sim B$ implies $A \stackrel{w k}{\sim} B$. Some authors, for example Greechie, use the phrase " $A$ and $B$ meet in the section $S_{e}$ " to describe $A \stackrel{w k}{\sim} B[4]$.

Definition 1.2. Let $L$ be an OML, and $A, B \in \mathfrak{A}_{L}$. We will say that $A$ and $B$ are path-connected in $L$, strictly path-connected in $L$ if $A$ and $B$ are joined 
by a proper path, a strictly proper path, respectively. An OML $S$ is not pathconnected if there exist two blocks in $L$ which are not path-connected. An OML $L$ is path-connected in $L$, strictly path-connected in $L$ if any two blocks in $L$ are joined by a proper path, a strictly proper path, respectively. An OML $L$ is called relatively path-connected if and only if each $[0, x]$ is path-connected for all $x \in L$.

Let $L$ be an OML, and $A, B, C \in \mathfrak{A}_{L}$. If $A$ and $B$ are joined with a strictly proper path $A=B_{0} \approx B_{1} \approx \cdots \approx B_{m-1} \approx B_{m}=B$ and if $B$ and $C$ are joined with a strictly proper path $B=C_{0} \approx C_{1} \approx \cdots \approx C_{n-1} \approx C_{n}=C$ then $A$ and $C$ are strictly path-connected by the concatenated path $A=B_{0} \approx B_{1} \approx \cdots \approx$ $B_{m-1} \approx B \approx C_{1} \approx \cdots \approx C_{n-1} \approx C_{n}=C$.

The following lemma is well known [1].

Lemma 1.3. If $L_{1}, L_{2}$ are OMLs, $L=L_{1} \times L_{2}, A, B \in \mathfrak{A}_{L_{1}}$ and $C, D \in \mathfrak{A}_{L_{2}}$, then $A \times C \sim B \times D$ holds in $L$ if and only if either $A=B$ and $C \sim D$ or $A \sim B$ and $C=D$. If $A$ and $B$ are linked at a then $A \times C$ and $B \times C$ are linked at $(a, 0)$. If $C$ and $D$ are linked at $c$ then $A \times C$ and $A \times D$ are linked at $(0, c)$.

The following four theorems are well known [7].

Theorem 1.4. Let $L$ be an $O M L$, and $x \in L$. Then $\mathbf{C}(x)$ is path-connected if and only if $[0, x]$ and $\left[0, x^{\prime}\right]$ are path-connected.

Proof. We know that $\mathbf{C}(x)=[0, x] \oplus\left[0, x^{\prime}\right]$. First, if $[0, x]$ and $\left[0, x^{\prime}\right]$ are pathconnected, then $\mathbf{C}(x)$ is path-connected by Lemma 1.3. Conversely, assume that $\mathbf{C}(x)$ is path-connected and let us prove that $[0, x]$ and $\left[0, x^{\prime}\right]$ are pathconnected. It is sufficient to show that $[0, x]$ is path-connected by symmetry. Let $A, B$ be distinct blocks in $[0, x]$ and let $D \in \mathfrak{A}_{\left[0, x^{\prime}\right]}$. We may assume that $A \cup B \not{Z}[0, x]$, otherwise $A$ and $B$ are path-connected in $[0, x]$. Then $A \oplus D$ and $B \oplus D$ are blocks in $\mathbf{C}(x)$ and hence path-connected in $\mathbf{C}(x)$. Let $A \oplus D=C_{0} \oplus E_{0} \sim C_{1} \oplus E_{1} \sim \cdots \sim C_{n} \oplus E_{n}=B \oplus D(n \geq 2)$ be a path joining $A \oplus D$ and $B \oplus D$ in $\mathbf{C}(x)$ where $C_{i} \in \mathfrak{A}_{[0, x]}$ and $E_{i} \in \mathfrak{A}_{\left[0, x^{\prime}\right]}$ $\forall(0 \leq i \leq n)$. Then the sequence $C_{0}, C_{1}, \ldots, C_{n}$ satisfies $C_{i} \sim C_{i+1}$ in $[0, x]$ or $C_{i}=C_{i+1}$ by Lemma 1.3. Let $M=\left\{i \mid C_{i} \sim C_{i+1}, 1 \leq i \leq n-1\right\}$. Then $A=C_{0} \sim C_{i_{1}} \sim \cdots \sim C_{i_{k}} \sim C_{n}=B$ where $i_{j} \in M$ such that $0=i_{0} \leq i_{1} \leq$ $i_{2} \leq \cdots \leq i_{k} \leq n-1$. Thus $A$ and $B$ are path-connected in $[0, x]$, and hence $[0, x]$ is path-connected. This completes the proof.

Theorem 1.5. Every finite direct product of path-connected orthomodular lattices is path-connected.

Theorem 1.6. Every infinite direct product of path-connected OMLs containing infinitely many non-Boolean factors is not path-connected.

Theorem 1.7. Let $L$ be an $O M L$. Then the following are equivalent:

(1) $L$ is relatively path-connected; 
(2) $\mathbf{C}(x)$ is path-connected $\forall x \in L$;

(3) $S_{x}$ is path-connected $\forall x \in L$.

\section{Path-connected orthomodular lattices}

$A$ site is a subalgebra of an OML $L$ of the form $S=A \cap B$, where $A$ and $B$ are distinct blocks of $L$. An OML $L$ is called with uniformly finite sites if there exists a natural number $n$ such that for all distinct blocks $A, B$ of $L$, $|A \cap B|<n$. An OML $L$ is called with finite sites if for all distinct blocks $A, B$ of $L,|A \cap B|<\infty$.

Using the pasting suggested by Greechie [4] and the inductive limit introduced by Dacey [3], we will present an OML with finite sites which is not path-connected.

A sublattice $M$ of an OML $L$ is said to be a suborthomodular lattice of $L$ in case the restriction of the orthocomplementation on $L$ makes $M$ an OML. A suborthomodular lattice $M$ of an OML $L$ is called subcomplete in case $N \subset M$ and $\bigvee N$ exists as computed in $L$ implies $\bigvee N$ is in $M$.

In what follows we assume that $\left(L_{1}, \leq_{1},{ }^{\sharp}\right)$ and $\left(L_{2}, \leq_{2},{ }^{+}\right)$are two disjoint OMLs, that $S^{i}$ is a proper suborthomodular lattice of $L_{i}(i=1,2)$, and that there exists an orthoisomorphism $\theta: S^{1} \rightarrow S^{2}$.

Definition 2.1. (1) Let $L_{0}=L_{1} \cup L_{2}$.

(2) Let $P_{1}=\left\{(x, y) \in L_{0} \times L_{0}: y=x \theta\right\}$.

(3) Let $\Delta=\left\{(x, x): x \in L_{0}\right\}$.

(4) Let $P$ be the equivalence relation defined by $P=\Delta \cup P_{1} \cup P_{1}^{-1}$, where $P_{1}^{-1}=\left\{(y, x):(x, y) \in P_{1}\right\}$.

(5) Let $L=L_{0} / P$.

(6) For $i=1,2$, let $R=\left\{([x],[y]) \in L \times L\right.$ : there exist $x_{i} \in[x]$ and $y_{i} \in[y]$ such that $\left.x_{i}<_{i} y_{i}\right\}$

(7) Let $\leq$ be the relation $\left(R_{1} \cup R_{2}\right)^{2}$.

(8) Define $[0]$ to be $\left[0_{1}\right]$ and $[1]$ to be $\left[1_{1}\right]$, where $0_{1}$ and $1_{1}$ are the zero and unit elements of $L_{1}$.

(9) Define ' $: L \rightarrow L$ by the following prescription: for $[x] \in L$, $[x]^{\prime}= \begin{cases}{\left[x_{1}^{\sharp}\right],} & \text { if there exists } x_{1} \in L_{1} \text { such that } x_{1} \in[x], \\ {\left[x_{2}{ }^{+}\right],} & \text {if there exists } x_{2} \in L_{2} \text { such that } x_{2} \in[x] .\end{cases}$

(10) Two sections $S^{1}$ and $S^{2}$ are said to be corresponding sections of $L_{1}$ and $L_{2}$ in case there exists $M_{i} \subset S^{i} \subset L_{i}(i=1,2)$ such that $M_{1} \theta=M_{2}$ and $S^{1}=\bigcup\left\{S_{m^{\sharp}}: m \in M_{1}\right\}$ and $S^{2}=\bigcup\left\{S_{m^{+}}: m \in M_{2}\right\}$.

The following theorem is well known [4].

Theorem 2.2 ([4]). Let $S^{1}$ and $S^{2}$ be corresponding sections of $L_{1}$ and $L_{2}$. Let $L_{i}$ be complete and let $S^{i}$ be subcomplete $(i=1,2)$. Then $L$ is a complete $O M L$. 
Definition 2.3. An OML $L$ is said to be obtained by pasting two OMLs $L_{1}$ and $L_{2}$ along the sections $S^{1}$ and $S^{2}$ if and only if all the conditions of Definition 2.1 are satisfied, and we write

$$
L=P\left(L_{1}, L_{2} ; S^{1}, S^{2} ; \theta\right) .
$$

A poset $D$ is called directed if any two elements subset of $D$ has an upper bound in $D$.

Definition 2.4. An inductive system $\left(\mathcal{A}_{\alpha}, \phi_{\beta}^{\alpha}\right)_{D}$ of sets is defined to be a triplet of the following objects:

(1) a directed partially ordered set $(D, \leq)$;

(2) sets $\mathcal{A}_{\alpha}$ for each $\alpha \in D$;

(3) mappings $\phi_{\beta}^{\alpha}$ for all $\alpha \leq \beta$, where $\phi_{\beta}^{\alpha}$ maps $\mathcal{A}_{\alpha}$ into $\mathcal{A}_{\beta}$ such that $\phi_{\gamma}^{\beta} \phi_{\beta}^{\alpha}=\phi_{\gamma}^{\alpha}$ for $\alpha \leq \beta \leq \gamma$ and $\phi_{\alpha}^{\alpha}$ is the identity mapping for all $\alpha \in D$.

$A \operatorname{limit}\left(\mathcal{A}, \phi^{\alpha}\right)$ of an inductive system (or an inductive limit) is a set $\mathcal{A}$ together with mappings $\phi^{\alpha}: \mathcal{A}_{\alpha} \rightarrow \mathcal{A}$, subject to the following conditions: $\phi^{\beta} \phi_{\beta}^{\alpha}=\phi^{\alpha}$ for $\alpha \leq \beta$ and, if mapping $\psi_{\alpha}: \mathcal{A}_{\alpha} \rightarrow \mathcal{B}$ are given with $\psi_{\beta} \phi_{\beta}^{\alpha}=\psi_{\alpha}$ for $\alpha \leq \beta$, then there exists a unique $\psi: \mathcal{A} \rightarrow \mathcal{B}$ such that $\psi_{\alpha}=\psi \phi^{\alpha}$ for $\alpha \in D$.

Let $(D, \leq)$ be a directed set. Assume that for each $\alpha \in D, \mathcal{A}_{\alpha}$ is an OMP and for $\alpha \leq \beta$, there is an ortho-embedding $\phi_{\beta}^{\alpha}: \mathcal{A}_{\alpha} \rightarrow \mathcal{A}_{\beta}$ such that the family $\left(\phi_{\beta}^{\alpha}\right)$ satisfies $\phi_{\alpha}^{\alpha}=I d_{\alpha}, \phi_{\gamma}^{\beta} \circ \phi_{\beta}^{\alpha}=\phi_{\gamma}^{\alpha}$ for $\alpha \leq \beta \leq \gamma$. Then $\left(\mathcal{A}_{\alpha}, \phi_{\beta}^{\alpha}\right)_{D}$ is an inductive system in the category of OMPs and ortho-embeddings.

Let $\left(\mathcal{A}_{\alpha}, \phi_{\beta}^{\alpha}\right)_{D}$ be a fixed inductive system in the category of OMPs and ortho-embeddings. Let $X=\bigcup_{\alpha \in D} \mathcal{A}_{\alpha}$. Define a relation $\simeq$ on $X$ by: $x \simeq y, x \in$ $\mathcal{A}_{\alpha}, y \in \mathcal{A}_{\beta}$ if there exists $\gamma \in D$ such that $\alpha \leq \gamma, \beta \leq \gamma$ and $\phi_{\gamma}^{\alpha}(x)=\phi_{\gamma}^{\beta}(y)$. Then $\simeq$ is an equivalence relation on $X$ [3]. Let $\bar{x}=\{y \in X \mid y \simeq x\}$ and let $\mathcal{O}=\{\bar{x} \mid x \in X\}$. Define an ordering $\leq$ on $\mathcal{O}$ by: $\bar{x} \leq \bar{y}$ if there exist $\alpha \in D$, $x_{\alpha} \in \bar{x} \cap \mathcal{A}_{\alpha}$ and $y_{\alpha} \in \bar{y} \cap \mathcal{A}_{\alpha}$ such that $x_{\alpha} \leq y_{\alpha}$ in $\mathcal{A}_{\alpha}$. For $\alpha \in D$, let $1=\overline{1_{\alpha}}$, $0=\overline{0_{\alpha}}$ and define $\overline{x_{\alpha}}{ }^{\prime}=\overline{x_{\alpha}{ }^{\prime}}$. Then ${ }^{\prime}$ is an orthocomplementation on $\mathcal{O}$ [3].

The following Theorem 2.5 and Corollary 2.6 are well known [3].

Theorem 2.5 ([3]). If $\left(\mathcal{A}_{\alpha}, \phi_{\beta}^{\alpha}\right)_{D}$ is an inductive system in the category of OMPs and ortho-embeddings, then its inductive limit exists in the same category and equals $\left(\mathcal{O}, \phi^{\alpha}\right)$, where $\mathcal{O}$ is in the above, and $\phi^{\alpha}: \mathcal{A}_{\alpha} \rightarrow \mathcal{O}$ is defined by $\phi^{\alpha}\left(x_{\alpha}\right)=\overline{x_{\alpha}}$.

Corollary 2.6. The inductive limit $\mathcal{O}$ of an inductive system of OMPs is $O M L$ if and only if $\mathcal{A}_{\alpha}$ is an $O M L$.

Let $X=\left\{a_{1}, a_{2}, a_{3}, \ldots\right\}$, and let $\wp(X)$ be the power set of $X$. Then the Boolean algebra $B$ consists of all finite and cofinite elements of the power set $\wp(X)$ of $X$ is denoted by

$$
B=\left\langle a_{1}, a_{2}, a_{3}, \ldots\right\rangle .
$$


The pasting of two disjoint OMLs $L_{1}$ and $L_{2}$ along the principal sections $S_{c_{1}} \leq$ $L_{1}$ and $S_{c_{2}} \leq L_{2}$ generated by $c_{1}, c_{2}$ respectively is denoted by

$$
L=P\left(L_{1}, L_{2} ; S_{c_{1}}, S_{c_{2}} ; \theta\right)
$$

(see Definition 2.3). We may omit the isomorphism $\theta$ if there is no difficulty.

Let $X=\left\{b_{i j}^{k} \mid 1 \leq i, k<\omega, 1 \leq j \leq 4\right\} \cup\left\{c_{3}, c_{4}\right\}$ be such that $b_{i j}^{k} \neq b_{m n}^{l}$ unless $(i, j, k)=(m, n, l)$, and $c_{3} \neq c_{4}$ and $c_{3}, c_{4} \neq b_{i j}^{k} \forall i, j, k$. In the following construction each $\left[b_{i j}^{i}\right]$ represents equivalent class containing $b_{i j}^{i} \forall i, j$ which have been defined. Now we are ready to present an OML $\mathfrak{L}$ with finite sites which is not path-connected (Figure 1).

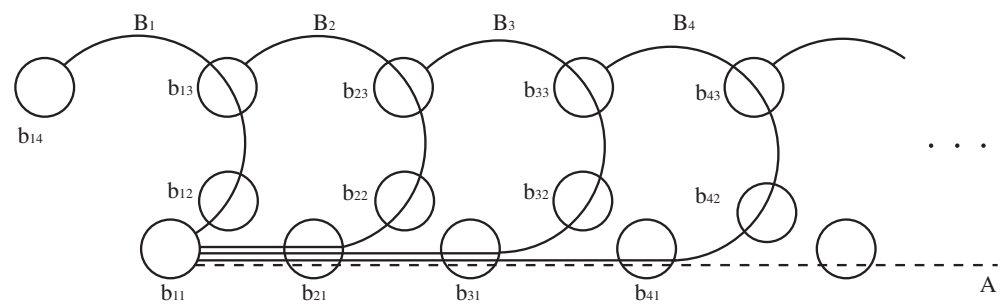

Figure 1. Greechie Diagram of the OML $\mathfrak{L}$ in Theorem 2.9

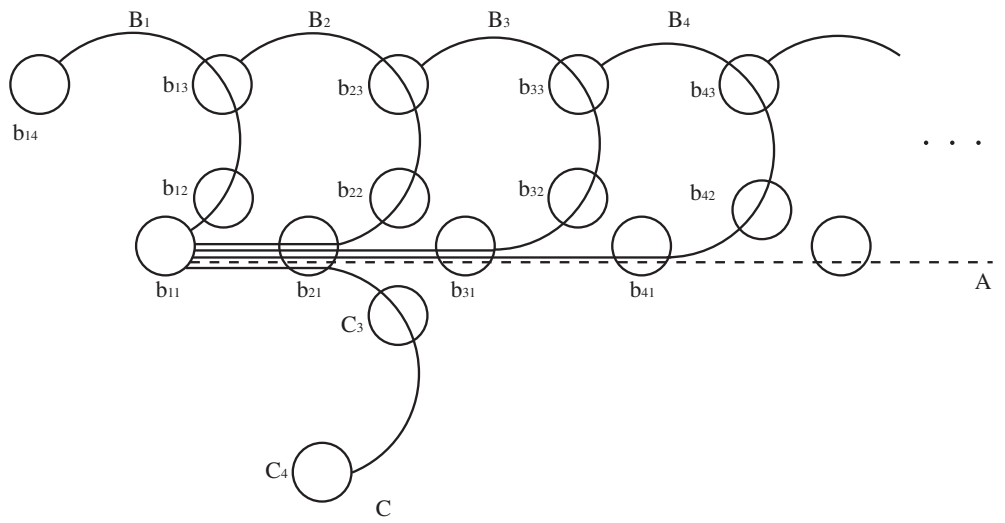

Figure 2. Greechie Diagram of the OML $\mathfrak{L}_{0}$ in Theorem 2.11

In the above two figures, the bars labeling each element and each block are omitted in order to make the diagrams simple; thus $b_{i j}\left(B_{i}\right)$ represents $\overline{b_{i j}}\left(\overline{B_{i}}\right)$.

Let $B_{1}=\left\langle b_{11}^{1}, b_{12}^{1}, b_{13}^{1}, b_{14}^{1}\right\rangle, B_{2}=\left\langle b_{11}^{2}, b_{21}^{2}, b_{22}^{2}, b_{23}^{2}, b_{13}^{2}\right\rangle, B_{3}=\left\langle b_{11}^{3}, b_{21}^{3}, b_{31}^{3}\right.$, $\left.b_{32}^{3}, b_{33}^{3}, b_{23}^{3}\right\rangle, \ldots$, and $B_{n}=\left\langle b_{11}^{n}, b_{21}^{n}, \ldots, b_{n 1}^{n}, b_{n 2}^{n}, b_{n 3}^{n}, b_{(n-1) 3}^{n}\right\rangle$. 
We construct $L_{n}(1 \leq n<\omega)$ by induction (Figure 1). Let $L_{1}=B_{1}$. Then $L_{1}$ is an OML. Let $L_{2}=P\left(L_{1}, B_{2} ; S_{e_{1}}, S_{e_{1}^{2}} ; \theta_{1}\right)$, where $e_{1}=\left(b_{11}^{1} \vee b_{13}^{1}\right)^{\prime}$, $e_{1}^{2}=\left(b_{11}^{2} \vee b_{13}^{2}\right)^{\prime}$ and $\theta_{1}$ is induced by the mapping sending $b_{i j}^{1}$ in $L_{1}$ to $b_{i j}^{2}$ in $B_{2}$ for $(i, j) \in\{(1,1),(1,3)\}$. Then $L_{2}$ is an OML by Theorem 2.2. Let $\left[b_{i j}^{2}\right]$ be the equivalent class in $L_{2}$ containing $b_{i j}^{k} \forall i, j, k$ such that $1 \leq i, k \leq 2$ and $1 \leq j \leq 4$. Note that $b_{i j}^{2}$ is equivalent to $b_{i j}^{1}$. Let $L_{3}=P\left(L_{2}, B_{3} ; S_{e_{2}}, S_{e_{2}^{3}} ; \theta_{2}\right)$, where $e_{2}=\left(\left[b_{11}^{2}\right] \vee\left[b_{21}^{2}\right] \vee\left[b_{23}^{2}\right]\right)^{\prime}, e_{2}^{3}=\left(b_{11}^{3} \vee b_{21}^{3} \vee b_{23}^{3}\right)^{\prime}$ and $\theta_{2}$ is induced by the mapping sending $\left[b_{i j}^{2}\right]$ in $L_{2}$ to $b_{i j}^{3}$ in $B_{3}$ for $(i, j) \in\{(1,1),(2,1),(2,3)\}$. Then $L_{3}$ is an OML by Theorem 2.2. Let $\left[b_{i j}^{3}\right]$ be the equivalent class in $L_{3}$ containing $b_{i j}^{k} \forall i, j, k$ such that $1 \leq i, k \leq 3$ and $1 \leq j \leq 4$. Note that $b_{i j}^{3}$ is equivalent to $b_{i j}^{k} \forall k$ such that $k<3 . L_{4}=P\left(L_{3}, B_{4} ; S_{e_{3}}, S_{e_{3}^{4}} ; \theta_{3}\right)$, where $e_{3}=\left(\left[b_{11}^{3}\right] \vee\left[b_{21}^{3}\right] \vee\left[b_{31}^{3}\right] \vee\left[b_{33}^{3}\right]\right)^{\prime}, e_{3}^{4}=\left(b_{11}^{4} \vee b_{21}^{4} \vee b_{31}^{4} \vee b_{33}^{4}\right)^{\prime}$ and $\theta_{3}$ is induced by the mapping sending $\left[b_{i j}^{3}\right]$ in $L_{3}$ to $b_{i j}^{4}$ in $B_{4}$ for $(i, j) \in\{(1,1),(2,1),(3,1),(3,3)\}$. Then $L_{4}$ is an OML by Theorem 2.2. Let $\left[b_{i j}^{4}\right]$ be the equivalent class in $L_{4}$ containing $b_{i j}^{k} \forall i, j, k$ such that $1 \leq i, k \leq 4$ and $1 \leq j \leq 4$. Note that $b_{i j}^{4}$ is equivalent to $b_{i j}^{k} \forall k$ such that $k<4$. Assume that $L_{n-1}$ has been constructed. Let $\left[b_{i j}^{n-1}\right]$ be the equivalent class in $L_{n-1}$ containing $b_{i j}^{k} \forall i, j, k$ such that $1 \leq i, k \leq n-1$ and $1 \leq j \leq 4$. Note that $b_{i j}^{n-1}$ is equivalent to $b_{i j}^{k} \forall k$ such that $k<n-1$. Let $L_{n}=P\left(L_{n-1}, B_{n} ; S_{e_{(n-1)}}, S_{e_{(n-1)}^{n}} ; \theta_{n-1}\right)$, where $e_{(n-1)}=\left(\left(\bigvee_{i=1}^{n-1}\left[b_{i 1}^{(n-1)}\right]\right) \vee\left[b_{(n-1) 3}^{(n-1)}\right]\right)^{\prime}, e_{(n-1)}^{n}=\left(\left(\bigvee_{i=1}^{n-1} b_{i 1}^{n}\right) \vee b_{(n-1) 3}^{n}\right)^{\prime}$ and $\theta_{n-1}$ is induced by the mapping sending $\left[b_{i j}^{n-1}\right]$ in $L_{n-1}$ to $b_{i j}^{n}$ in $B_{n}$ for $(i, j) \in\{(1,1),(2,1), \ldots,((n-1), 1),((n-1), 3)\}$. Then $L_{n}$ is an OML by Theorem 2.2. Let $\left[b_{i j}^{n}\right]$ be an equivalent class in $L_{n}$ containing $b_{i j}^{k} \forall i, j, k$ such that $1 \leq i, k \leq n$ and $1 \leq j \leq 4$. Note that $b_{i j}^{n}$ is equivalent to $b_{i j}^{k} \forall k$ such that $k<n$.

Let $\phi_{j}^{i}$ be an ortho-embedding from $L_{i}$ into $L_{j} \forall i, j(1 \leq i \leq j<\omega)$. Then $\left(L_{i}, \phi_{j}^{i}\right)_{(1 \leq i \leq j<\omega)}$ is an inductive system in the category of orthomodular posets and ortho-embeddings.

Let $X=\bigcup_{(1<i<\omega)} L_{i}$. Define a relation $\simeq$ on $X$ by: $x \simeq y, x \in L_{i}$, $y \in L_{j}$ if and only if there exists $1 \leq k<\omega$ such that $1 \leq i \leq k<\omega$, $1 \leq j \leq k<\omega$ and $\phi_{k}^{i}(x)=\phi_{k}^{j}(y)$. Then $\simeq$ is an equivalence relation on $X$ [3]. Let $\bar{x}=\{y \in X \mid y \simeq x\}$ and let $\mathfrak{L}=\{\bar{x} \mid x \in X\}$ (Figure 1). Define an ordering $\leq$ on $\mathfrak{L}$ by: $\bar{x} \leq \bar{y}$ if and only if there exist $i, x_{i}$ and $y_{i}$ such that $1 \leq i<\omega$, $x_{i} \in \bar{x} \cap L_{i}, y_{i} \in \bar{y} \cap L_{i}$ and $x_{i} \leq y_{i}$ in $L_{i}$. For $1 \leq i<\omega$, let $1=\overline{1_{i}}, 0=\overline{0_{i}}$, and define ${\overline{x_{i}}}^{\prime}=\overline{x_{i}{ }^{\prime}}$. Then ' in an orthocomplementation on $\mathfrak{L}[3]$. Thus $\left(\mathfrak{L}, \phi^{i}\right)$ is the inductive limit of the inductive system $\left(L_{i}, \phi_{j}^{i}\right)_{1 \leq i \leq j<\omega}$, where $\phi^{i}: L_{i} \rightarrow \mathfrak{L}$ is defined by $\phi^{i}\left(x_{i}\right)=\overline{x_{i}}$ by Theorem 2.5. Moreover, $\mathfrak{L}$ is an OML by Corollary 2.6 since $L_{i}$ is an OML $\forall i(1 \leq i<\omega)$.

Lemma 2.7. $\mathfrak{A}_{\mathfrak{L}}=\left\{\overline{B_{i}} \mid 1 \leq i<\omega\right\} \cup\{A\}$. 
Proof. We know that $\left\{\overline{B_{i}} \mid 1 \leq i<\omega\right\} \subset \mathfrak{A}_{\mathfrak{L}}$ by our inductive construction. Let $\left[B^{i}\right],\left[C^{i}\right] \in \mathfrak{A}_{L_{i}}$. Then $\phi_{j}^{i}\left(\left[B^{i}\right]\right)=\left[B^{j}\right]$ for some $\left[B^{j}\right] \in \mathfrak{A}_{L_{j}} \forall i, j(1 \leq i \leq j<\omega)$ and $\phi_{j}^{i}\left(\left[B^{i}\right]\right) \neq \phi_{j}^{i}\left(\left[C^{i}\right]\right)$ if $\left[B^{i}\right] \neq\left[C^{i}\right]$.

Let $\left[B^{i}\right],\left[C^{i}\right] \in \mathfrak{A}_{L_{i}}$. Then $\phi^{i}\left(\left[B^{i}\right]\right)=\bar{B}$ for some $\bar{B} \in \mathfrak{A}_{\mathfrak{L}} \forall i(1 \leq i<\omega)$ and $\phi^{i}\left(\left[B^{i}\right]\right) \neq \phi^{i}\left(\left[C^{i}\right]\right)$ if $\left[B^{i}\right] \neq\left[C^{i}\right]$.

Therefore $\forall i(1 \leq i<\omega) \overline{B_{i}} \in \mathfrak{A}_{\mathfrak{L}}$, where $\overline{B_{i}}$ is the equivalent class containing $\left[B_{i}^{j}\right] \forall j(1 \leq j<\omega)$. Moreover, $\mathfrak{L}=\bigcup\left\{\bar{B} \mid B \in \mathfrak{A}_{L_{i}}\right\}$.

Let $A=\left\langle\overline{b_{11}}, \overline{b_{21}}, \overline{b_{31}}, \ldots, \overline{b_{n 1}}, \ldots\right\rangle$, and let $\bar{x}, \bar{y} \in A$. Then there exists $\overline{B_{i}} \in \mathfrak{A}_{\mathfrak{L}}$ such that $\bar{x}, \bar{y} \in \overline{B_{i}}$ and hence $\bar{x} \mathbf{C} \bar{y}$. Therefore $A$ is a commuting set. If $\bar{x} \notin \tilde{A}$, then by our construction there exists $\overline{B_{k}} \in \mathfrak{A}_{\mathfrak{L}}$ such that $\bar{x} \in \overline{B_{k}}$ and hence $\overline{b_{(k+2), 1}} \in \overline{B_{k+2}} \cap A$ with $\bar{x} \not \overline{b_{(k+2), 1}}$. Hence $A$ is a maximal commuting set. We claim that $\mathfrak{A}_{\mathfrak{L}}=\left\{\overline{B_{i}} \mid 1 \leq i<\omega\right\} \cup\{A\}$. Let $C \in \mathfrak{A}_{\mathfrak{L}}$. We may assume that $C \neq A$. Thus there exists an atom $\bar{z} \in C \backslash A$ and hence there exists $\overline{B_{h}} \in \mathfrak{A}_{\mathfrak{L}}$ such that $\bar{z} \in \overline{B_{h}}$. We have the following three cases: (1) there exists a unique $h$ such that $\bar{z} \in \overline{B_{h}}$ and hence $C=\overline{B_{h}} ;(2) \bar{z} \in \overline{B_{h}} \cap \overline{B_{h+1}}$ and hence $C=\overline{B_{h}}$ or $C=\overline{B_{h+1}}$; (3) $\bar{z} \in \overline{B_{h-1}} \cap \overline{B_{h}}$ and hence $C=\overline{B_{h-1}}$ or $C=\overline{B_{h}}$. This completes the proof.

An OML $L$ is called the horizontal sum of a family $\left(L_{i}\right)_{i \in I}$ (denoted by $\left.\circ\left(L_{i}\right)_{i \in I}\right)$ of at least two subalgebras, if $\bigcup L_{i}=L$, and $L_{i} \cap L_{j}=\{0,1\}$ whenever $i \neq j$, and one of the following equivalent conditions is satisfied:

(1) if $x \in L_{i} \backslash L_{j}$ and $y \in L_{j} \backslash L_{i}$, then $x \vee y=1$;

(2) every block of $L$ belongs to some $L_{i}$;

(3) if $S_{i}$ is a subalgebra of $L_{i}$, then $\bigcup S_{i}$ is a subalgebra of $L$ [2].

An OML $L$ is said to be the weak horizontal sum of a family $\left(L_{i}\right)_{i \in I}$ of subalgebras if and only if there exists an isomorphism $f$ of $L$ onto a product of $L_{0} \times L^{\prime}$ of a Boolean algebra $L_{0}$ and an OML $L^{\prime}$ such that the subalgebras $L_{i}$ of $L$ correspond via $f$ to subalgebras of the form $L_{0} \times L_{i}^{\prime}$ and $L^{\prime}$ is the horizontal sum of the family $\left(L_{i}^{\prime}\right)_{i \in I}[1]$.

In the following Lemma 2.8, Theorem 2.9, Lemma 2.10 and Theorem 2.11, the bars labeling each element and each block are omitted in order to make the notation simple; thus $b_{i j}$ and $B_{i}$ represent $\overline{b_{i j}}$ and $\overline{B_{i}}$, respectively.

Lemma 2.8. Two blocks of the type $B_{i}$ and $B_{j}(1 \leq i \leq j<\omega)$ in $\mathfrak{L}$ have the following properties: $B_{i} \cup B_{j} \leq \mathfrak{L}$ if $j=i+1$ or $j \geq i+4$, and $B_{i} \cup B_{j} \not \leq \mathfrak{L}$ if $j=i+2, i+3$.

Proof. We know that $B_{i} \cup B_{j} \leq \mathfrak{L}$ if $j=i+1$ or $j \geq i+4$ since $B_{i} \cup B_{j}$ is a weak horizontal sum of $B_{i}$ and $B_{j}$. Let us prove that $B_{i} \cup B_{j} \not{L} \mathfrak{L}$ if $j=i+2$ or $i+3$.

Let $x=b_{11} \vee b_{21} \vee \cdots \vee b_{(i+1) 1} \vee b_{(i+1) 3} \in B_{i+2}$. Then $x \vee b_{i 3}=b_{(i+1) 2}^{\prime} \in B_{i+1}$ and $x \vee b_{i 3}=b_{(i+1) 2}^{\prime} \notin \mathfrak{L} \backslash B_{i+1}$. Thus $B_{i} \cup B_{i+2} \not \leq \mathfrak{L}$. 
Let $y=b_{11} \vee b_{21} \vee \cdots \vee b_{(i+2) 1} \vee b_{(i+2) 3} \in B_{i+3}$. Then $y \vee b_{i 3}=b_{(i+1) 3}^{\prime} \in$ $B_{i+1} \cap B_{i+2}$ and $y \vee b_{i 3}=b_{(i+1) 3}^{\prime} \notin \mathfrak{L} \backslash\left(B_{i+1} \cup B_{i+2}\right)$. Thus $B_{i} \cup B_{i+3} \not \leq \mathfrak{L}$.

We know that every OML with uniformly finite sites is path-connected [8]. The following theorem shows that there exists an OML with finite sites which is not path-connected.

Theorem 2.9. There exists a weakly path-connected OML with finite sites which is not path-connected.

Proof. Let $A=\left\langle b_{11}, b_{21}, b_{31}, \ldots, b_{n 1}, \ldots\right\rangle$. Then $\mathfrak{A}_{\mathfrak{L}}=\{A\} \cup\left\{B_{i} \mid 1 \leq i<\omega\right\}$ by Lemma 2.7 .

First, let us prove that $\mathfrak{L}$ is with finite sites. Since $\left|A \cap B_{i}\right|=\left|S_{f_{i}}\right|=2^{i+1}$, where $f_{i}=\left(\bigvee_{k=1}^{i} b_{k 1}\right)^{\prime}$ and $\left|B_{i} \cap B_{j}\right| \leq 2^{i+1} \forall(1 \leq i \leq j<\omega), \mathfrak{L}$ is an OML with finite sites.

Second, let us prove that $A$ is not path-connected with any $B_{i} \in \mathfrak{A}_{\mathfrak{L}} \forall i(1 \leq$ $i<\omega)$. Fix such $i$ and let $x=\bigvee_{1<k<i+2} b_{k 1} \in A$, and let $y=\tilde{b}_{i 3} \in B_{i}$. Then $x \vee y=b_{(i+1) 3}^{\prime} \notin A \cup B_{i}$. Thus $A \cup B_{i} \not \leq \mathfrak{L} \quad \forall i(1 \leq i<\omega)$ since $\mathfrak{A}_{\mathfrak{L}}=\{A\} \cup\left\{B_{i} \mid 1 \leq i<\omega\right\}$. Hence $\mathfrak{L}$ is not path-connected since $A$ is not path-connected with any other block of $L$ except itself.

Finally, let us prove that $\mathfrak{L}$ is weakly path-connected. $B_{i}$ and $B_{j}$ are pathconnected for all $1 \leq i<j<\omega$ by a path $B_{i} \sim B_{i+1} \sim \cdots \sim B_{j}$ by Lemma 2.8 and hence weakly path-connected. $A$ is weakly path-connected with $B_{i}$ since $A \cap B_{i}=S_{f_{i}} \cap\left(A \cup B_{i}\right) \quad(1 \leq i<\omega)$, where $f_{i}=\left(\bigvee_{k=1}^{i} b_{k 1}\right)^{\prime}$. Therefore $\mathfrak{L}$ is weakly path-connected.

Let $\left\{\widetilde{b}_{11}, \widetilde{b}_{21}\right\} \cap\left\{\overline{b_{i j}} \mid \overline{b_{i j}} \in \mathfrak{L}\right\}=\emptyset$ and $C=\left\langle\widetilde{b}_{11}, \widetilde{b}_{21}, c_{3}, c_{4}\right\rangle$. Let $\mathfrak{L}_{0}=$ $P\left(\mathfrak{L}, C ; S_{\left(\overline{b_{11}} \vee \overline{b_{21}}\right)^{\prime}}, S_{\left.\widetilde{(b}_{11} \vee \widetilde{b}_{21}\right)^{\prime}} ; \theta\right)$, where $\theta$ is induced by the map sending $\overline{b_{i j}}$ to $\widetilde{b}_{i j}$ for $(i, j) \in\{(1,1),(2,1)\}$ (Figure 2 ). Then $\mathfrak{L}_{0}$ is an OML by Theorem 2.2 and $\mathfrak{A}_{\mathfrak{L}_{0}} \cong \mathfrak{A}_{\mathfrak{L}} \cup C$.

Lemma 2.10. Every $\mathbf{C}\left(b_{i 1}\right)(1<i<\omega)$ in $\mathfrak{L}$ is isomorphic to $2^{i-1} \times \mathfrak{L}$, and every $\mathbf{C}\left(b_{i 1}\right)(2<i<\omega)$ in $\mathfrak{L}_{0}$ is $\mathfrak{L}$ is isomorphic to $2^{i-1} \times \mathfrak{L}$.

Proof. We know that $\mathbf{C}\left(b_{i 1}\right)=\mathbf{C}\left(b_{i 1}\right)\left[0, \bigvee_{k=1}^{i-1} b_{k 1}\right] \oplus \mathbf{C}\left(b_{i 1}\right)\left[0,\left(\bigvee_{k=1}^{i-1} b_{k 1}\right)^{\prime}\right]$.

Moreover, $\mathbf{C}\left(b_{i 1}\right)\left[0, \bigvee_{k=1}^{i-1} b_{k 1}\right] \cong 2^{i-1}$, and $\mathbf{C}\left(b_{i 1}\right)\left[0,\left(\bigvee_{k=1}^{i-1} b_{k 1}\right)^{\prime}\right] \cong \mathfrak{L}$ with the isomorphism $\phi: \mathbf{C}\left(b_{i 1}\right)\left[0,\left(\bigvee_{k=1}^{i-1} b_{k 1}\right)^{\prime}\right] \rightarrow \mathfrak{L}$ induced by $\phi\left(b_{k j}\right)=b_{(k-i+1) j}$ $\forall k$ such that $i \leq k$, i.e., $\phi\left(L_{j}\left[0,\left(\bigvee_{k=1}^{i-1} b_{k 1}\right)^{\prime}\right]\right)=L_{j-i+1} \forall j \geq i$. Similarly, every $\mathbf{C}\left(b_{i 1}\right)$ in $\mathfrak{L}_{0}$ is isomorphic to $2^{i-1} \times \mathfrak{L}$. This completes the proof.

Let $L$ be an OML. A subalgebra $S$ of $L$ is said to be a full subalgebra if every blocks of $S$ is a block of $L$. Note that $\mathbf{C}(x)$ is a full subalgebra of $L$ for all $x \in L$ since $\mathfrak{A}_{\mathbf{C}(x)}=\left\{B \in \mathfrak{A}_{L} \mid x \in B\right\}$.

Theorem 2.11. There exists a path-connected $O M L$ such that $\mathbf{C}(x)$ is not path-connected for some $x \in L$. 
Proof. First, let us show that $\mathfrak{L}_{0}$ is path-connected. $A \cup C \leq \mathfrak{L}_{0}$ and $A \cap C=$ $S_{\left(b_{11} \vee b_{21}\right)^{\prime}} \neq \mathbf{C}\left(\mathfrak{L}_{0}\right)=\left\{0, b_{11}, b_{11}^{\prime}, 1\right\}$ and hence $A \approx C$. Then $A$ is strictly path-connected with each $B_{i}$ since $A \approx C \approx B_{i}(1<i<\omega)$, and $B_{i} \sim C$ for all $1 \leq i \leq \omega$. Thus $\mathfrak{L}_{0}$ is path-connected.

Finally, $\mathbf{C}\left(b_{31}\right)$ is a full subalgebra of $\mathfrak{L}_{0}$ which is not path-connected since $\mathbf{C}\left(b_{31}\right) \cong 2^{2} \times \mathfrak{L}$ by Lemma 2.10 , and $\mathfrak{A}_{\mathbf{C}\left(b_{31}\right)}=\left\{B \in \mathfrak{A}_{\mathfrak{L}_{0}} \mid b_{31} \in B\right\}=$ $\{A\} \cup\left\{B_{i} \in \mathfrak{L} \mid B_{i}, 3 \leq i<\omega\right\}$. Thus $A \in \mathfrak{A}_{\mathbf{C}\left(b_{31}\right)}$ is not path-connected in $\mathbf{C}\left(b_{31}\right)$ with each $B_{i} \forall(3 \leq i<\omega)$, by the proof in Theorem 2.9. This completes the proof. OMLs.

We need the following Theorem 2.12 [5] to get a class of path-connected

Theorem $2.12([5])$. Let $L$ be an $O M L$. Then the set $\mathbf{C A}(L)$ of all central Abelian elements of $L$ is the set of orthocomplements of the upper bounds for the set $C o m L$, and $\bigvee \mathbf{C A}(L)$ exists if and only if $\bigvee$ Com $L$ exists. If $h=\bigvee \operatorname{Com} L$ exists, then $\mathbf{C A}(L)=\left[0, h^{\prime}\right]$ and $[0, h]$ contains no nonzero elements which are central Abelian elements of $[0, h]$ (and, therefore, of $L)$.

We denote the join-semilattice generated by $M \subset L$ of a lattice $L$ by $[M]_{\vee}$. $[M]_{\vee}$ consists of all $\bigvee M_{0}$ with $M_{0}$ a finite subset of $M$. Then we have the following structure theorem.

Theorem 2.13. If $L$ is a non-Boolean OML such that the height of the joinsemilattice $[C o m L]_{\vee}$ generated by the commutators of $L$ is finite, then $L$ has a unique orthogonal decomposition $L=\left[0, e_{0}\right] \oplus\left[0, e_{1}\right] \oplus \cdots \oplus\left[0, e_{n}\right]$, where $e_{0}$ is the largest central Abelian element of $L$, and each $\left[0, e_{i}\right](1 \leq k \leq n)$ is an irreducible non-Boolean OML such that the height of the join-semilattice $\left[\text { Com }\left[0, e_{i}\right]\right]_{\vee}$ generated by the commutators of $\left[0, e_{i}\right]$ is finite.

Proof. Let $L$ be a non-Boolean OML such that the height of the join-semilattice $[C o m L]_{\vee}$ generated by the commutators of $L$ is finite. Then $\bigvee$ Com $L$ exists. Let $e_{0}^{\prime}=\bigvee \operatorname{Com} L$. Since $e_{0}$ is central, $L=\left[0, e_{0}\right] \oplus\left[0, e_{0}^{\prime}\right]$. Thus $\operatorname{Com} L=$ $\operatorname{Com}\left[0, e_{0}^{\prime}\right]$ by Theorem 2.12. If $\left[0, e_{0}^{\prime}\right]=\bigoplus_{i \in I}\left[0, e_{i}\right]$ with each $e_{i}>0$, then each summand has at least two commutators since each $\left[0, e_{i}\right]$ is a non-Boolean OML and hence the height $h\left(\left[\operatorname{Com}\left[0, e_{i}\right]\right]_{\vee}\right) \geq 1$. We may assume that $I$ has the maximal cardinality among all such decompositions of $\left[0, e_{0}^{\prime}\right]$. Then $|I|<\infty$ and each interval $\left[0, e_{i}\right]$ is irreducible. Moreover, each $e_{i}(i \geq 1)$ is an atom of $\mathbf{C}(L)$. Since any such decomposition of $\left[0, e_{0}^{\prime}\right]$ is determined by the atoms of $\mathbf{C e n}\left(\left[0, e_{0}^{\prime}\right]\right)$, the decomposition is unique.

We need the following Lemma 2.14 to prove Theorem 2.15.

Lemma 2.14. Let $L$ be an $O M L$, and $A, B \in \mathfrak{A}_{L}$. If $A \cap B=\mathbf{C}(L)$ and $A \cup B \not L$, then there exist $C, D \in \mathfrak{A}_{L}$ such that $A \cap C \neq \mathbf{C}(L), C \cap D \neq \mathbf{C}(L)$ and $D \cap B \neq \mathbf{C}(L)$. 
Proof. There exist $c, d$ such that $c, d \in A \cup B$ and $c \vee d \notin A \cup B$ since $A \cup B \not L L$. Hence $c \vee d \notin \mathbf{C}(L)=\bigcap \mathfrak{A}_{L}$. We may assume that $c \in A \backslash B$ and $d \in B \backslash A$. Therefore there exist $C, D \in \mathfrak{A}_{L}$ such that $c, c \vee d \in C$ and $d, c \vee d \in D$. Then $c, d, c \vee d \notin \mathbf{C}(L)$ with $c \in A \cap C, c \vee d \in C \cap D$ and $d \in D \cap B$.

We can find the following class of path-connected OMLs which contains all commutator-finite OMLs [5] and all block-finite OMLs [1]. This containment is proper as may be proved simply by considering any orthocomplemented projective plane.

Theorem 2.15. If $L$ is an $O M L$ such that the height of the join-semilattice $[C o m L]_{\vee}$ generated by the commutators of $L$ is finite, then $L$ is path-connected.

Proof. Let $L$ be an OML such that the height of the join-semilattice $[C o m L]_{\vee}$ generated by the commutators of $L$ is finite. Then we may assume that $L$ is irreducible by Theorem 2.13 and Theorem 1.5. Let us prove that $L$ is pathconnected by induction on the height $k$ of $[\operatorname{Com} L]_{\vee}$ (with the ordering inherited from $L$ ). If $k=0$, then $L$ is path-connected since $L$ is a Boolean algebra. Assume that the conclusion of the theorem is true for each OML such that the height of the join-semilattice generated by the commutators of that OML is less than or equal to $n-1$. If $k=n \geq 1$, then $L$ is not a Boolean algebra. Thus there exist two distinct blocks $A, B$ of $L$.

Assume first that $A \cap B \neq\{0,1\}=\mathbf{C}(L)$. Let $m \in A \cap B \backslash\{0,1\}$. If the height of the join-semilattice $[\operatorname{Com} \mathbf{C}(m)]_{\vee}$ generated by $\operatorname{Com} \mathbf{C}(m)$ is less than the height of the join-semilattice $[C o m L]_{\vee}$, then $A$ and $B$ are path-connected in $\mathbf{C}(m)$ and hence in $L$ by the inductive hypothesis. Thus we may assume that $h\left([\operatorname{Com} \mathbf{C}(m)]_{\vee}\right)=h\left([\operatorname{Com} L]_{\vee}\right)$. Suppose $\bigvee \operatorname{Com} \mathbf{C}(m)<\bigvee \operatorname{Com} L$. Then $h\left([\operatorname{Com} \mathbf{C}(m)]_{\vee}\right)<h\left([\operatorname{Com} L]_{\vee}\right)$ contradicting $h\left([\operatorname{Com} \mathbf{C}(m)]_{\vee}\right)=h\left([\operatorname{Com} L]_{\vee}\right)$. Hence $\bigvee \operatorname{Com} \mathbf{C}(m)=\bigvee \operatorname{Com} L=1$ since $L$ is irreducible. Thus $\mathbf{C}(m)$ has no nontrivial Boolean factors by Theorem 2.12. Therefore $\mathbf{C}(m)[0, m](=$ $[0, m])$ and $\mathbf{C}(m)\left[0, m^{\prime}\right]\left(=\left[0, m^{\prime}\right]\right)$ are non-Boolean. Then $[0, m],\left[0, m^{\prime}\right]$ are path-connected since $h\left([\operatorname{Com}[0, m]]_{\vee}\right)<h\left([\operatorname{Com} L]_{\vee}\right)$ and $h\left(\left[\operatorname{Com}\left[0, m^{\prime}\right]\right]_{\vee}\right)$ $<h\left([\operatorname{Com} L]_{\vee}\right)$ by the inductive hypothesis. Thus $\mathbf{C}(m)=[0, m] \oplus\left[0, m^{\prime}\right]$ is path-connected by Theorem 1.4. Therefore $A$ and $B$ are path-connected in the full subalgebra $\mathbf{C}(m)$ of $L$. Thus $A, B$ are path-connected in $L$.

Assume finally that $A \cap B=\{0,1\}$. If $A \cup B \leq L$, then $A$ and $B$ are pathconnected. If $A \cup B \not L L$, then there exist $C, D \in \mathfrak{A}_{L}$ such that $A \cap C \neq\{0,1\}$, $C \cap D \neq\{0,1\}$ and $D \cap B \neq\{0,1\}$ by Lemma 2.14. Thus $A$ and $B$ are path-connected with a concatenated path by the first case.

As a special case of Theorem 2.15, if $L$ is an OML such that $\alpha \vee \beta=1$ for any distinct commutators $\alpha, \beta \notin\{0,1\}$, then $L$ is path-connected. The fact that every commutator-finite OML is path-connected [2], is also a corollary of this theorem. 


\section{References}

[1] G. Bruns, Block-finite orthomodular lattices, Canad. J. Math. 31 (1979), no. 5, 961-985.

[2] G. Bruns and R. Greechie, Blocks and commutators in orthomodular lattices, Algebra Universalis 27 (1990), no. 1, 1-9.

[3] J. Dacey, Orthomodular spaces, University of Massachusetts, Ph. D. thesis, 1968.

[4] R. Greechie, On the structure of orthomodular lattices satisfying the chain condition, J. Combinatorial Theory 4 (1968), 210-218.

[5] R. Greechie and L. Herman, Commutator-finite orthomodular lattices, Order 1 (1985), no. 3, 277-284.

6] G. Kalmbach, Orthomodular Lattices, Academic Press, London, 1983.

[7] E. Park, Relatively path-connected orthomodular lattices, Bull. Korean Math. Soc. 31 (1994), no. 1, 61-72.

[8] Soc. 14 (1999), no. 1, 31-37.

[9] M. Roddy, An orthomodular analogue of the Birkhoff-Menger theorem, Algebra Universalis 19 (1984), no. 1, 55-60.

EUNSOON PARK

Department of Mathematics

SOONGSIL UNIVERSITY

SEOUL 156-743, KOREA

E-mail address: espark@ssu.ac.kr

WONHEE SONG

Department of Mathematics

Graduate SCHOOL

SOONGSIL UNIVERSITY

SEOUl 156-743, KoreA

E-mail address: songwonhee@ssu.ac.kr 